\title{
PRACTICAL MODEL AND DETECTION ALGORITHM FOR VALVE STICTION
}

\author{
Manabu Kano* Hiroshi Maruta* \\ Hidekazu Kugemoto ${ }^{* *}$ Keiko Shimizu ${ }^{* * *}$
}

\author{
* Kyoto University, Kyoto 606-8501, Japan \\ ** Sumitomo Chemical Co. Ltd., Niihama 792-8521, Japan \\ *** TOSHIBA Corporation, Yokohama 230-0051, Japan
}

\begin{abstract}
Stiction is the most common problem in pneumatic control valves, which are widely used in the process industry. Stiction causes fluctuation of process variables, which lowers productivity. Therefore, developing a method to detect stiction and distinguish it from other causes is crucial to help operators take an appropriate action for improving control performance. The present work proposes a valve stiction model and new stiction detection algorithms. Using only two parameters, the proposed model can describe the stiction phenomenon with sufficient accuracy. The usefulness of the proposed detection methods are demonstrated by comparing them with a conventional method. The proposed methods are shown to successfully detect valve stiction, distinguish it from bad tuning or disturbances, and quantify the degree of stiction, by using simulation data sets and real operation data sets of several chemical processes. Copyright (C) 2004 IFAC
\end{abstract}

Keywords: Control Valve, Stiction, Control Performance Monitoring, Fault Detection, Fault Diagnosis

\section{INTRODUCTION}

The variability of process variables makes it difficult to keep operating conditions close to their bound and thus causes excessive energy consumption. To maximize productivity, control systems should achieve their best performance. The poor control performance is caused not only by bad controller tuning but also by undesired characteristics of control valves. Among the many types of undesired characteristics of control valves, stiction is the most common problem in the process industry. Therefore, it is important to develop a practical method that can detect stiction and distinguish it from other causes including inadequate controller tuning.
In the present work, a valve stiction model is built to understand the stiction phenomenon and simulate it. There are two types of models: 1) a detailed physical model that formulates the stiction phenomenon as precisely as possible (Muller, 1994), and 2) a data-driven model that describes the relationship between a controller output and a valve position (Choudhury et al., 2004). Since a detailed physical model has numbers of unknown physical parameters, it is not only time-consuming but not practical to simulate an actual control valve by using such a model. A data-driven model, on the other hand, is useful because it has only a few parameters that are easy to identify and simple to understand. However, the data-driven model proposed by Choudhury et al. (2004) has several 
disadvantages. The most serious problem would be their assumption that the input signal is deterministic; throughout their paper they only investigate sinusoidal inputs. In fact, when the input signal is stochastic the model does not function well. Although it has been pointed out that valve stiction causes persistent fluctuation of a process variable, stiction does not always cause persistent fluctuation. A valve stiction model should therefore be able to cope with both deterministic and stochastic signals. The model developed here is an improved and extended version of the model proposed by Choudhury et al. (2004). The accuracy of the model is validated by comparing simulation results with real operation data of a chemical process.

Furthermore, new methods for detecting valve stiction are proposed. Although several methods have been proposed (e.g., Horch, 2000), they are based on the assumption that a controlled variable such as flow rate follows a predefined probability distribution, and thus their judgment is not reliable when the assumption is not satisfied. In addition, conventional methods cannot quantify the degree of stiction even if they can detect it. The usefulness of new valve stiction detection methods is demonstrated by applying them to simulation data generated by using the developed model and real operation data obtained from several chemical processes.

\section{VALVE STICTION MODEL}

In this section, the stiction phenomenon of a pneumatic control valve, which is widely used in the process industry, is modeled.

\subsection{Pneumatic Control Valve and Stiction}

The general structure of a pneumatic control valve is shown in Fig. 1. This valve is closed by elastic force and opened by air pressure. Flow rate is changed according to the plug position, which is determined by the balance between elastic force and air pressure. The plug is connected to the valve stem. The stem is moved against static or kinetic frictional force caused by packing, which is a sealing device to prevent leakage of process fluid. Smooth movement of the stem is impeded by excessive static friction. The valve position cannot be changed until the controller output overcomes static friction, and it is suddenly and considerably changed when the difference between elastic force and air pressure exceeds the maximum static frictional force.

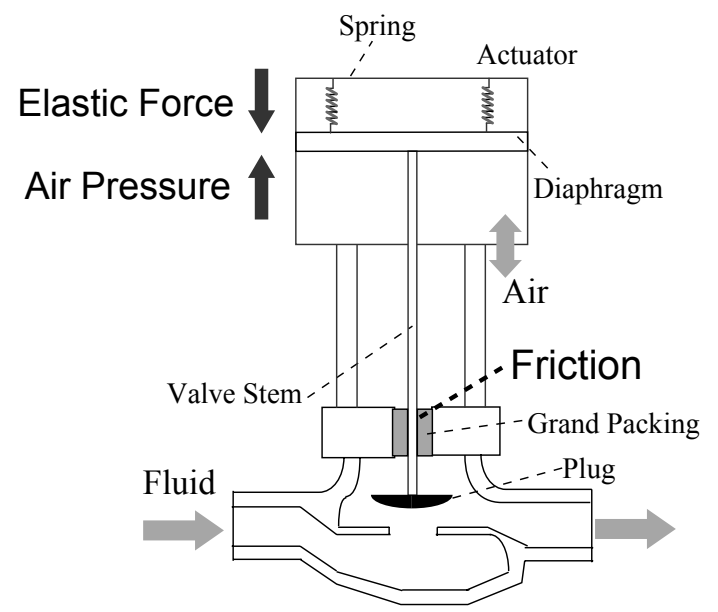

Fig. 1. Structure of pneumatic control valve

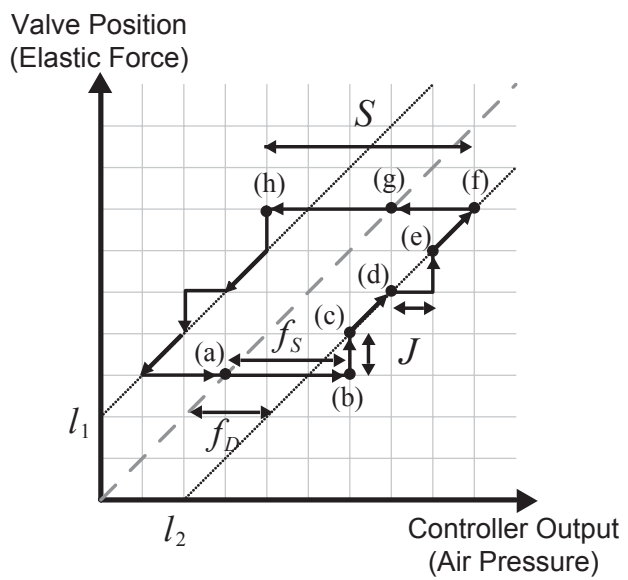

Fig. 2. Relation between controller output and valve position under valve stiction

\subsection{Stiction Model}

To model the relationship between the controller output and the valve position of a pneumatic control valve, the balance among elastic force, air pressure, and frictional force needs to be taken into account. The relationship can be described as shown in Fig. 2. The dashed line denotes the states where elastic force and air pressure are balanced. The controller output and the valve position change along this line in an ideal situation without any friction.

The ideal relationship is disturbed when friction arises. For example, the valve is resting at (a) where elastic force and air pressure are balanced. The valve position cannot be changed due to static friction even if the controller output, i.e., air pressure, is increased. The valve begins to open at (b) where the difference between air pressure and elastic force exceeds the maximum static frictional force. Since the frictional force changes from static $f_{S}$ to kinetic $f_{D}$ when the valve starts to move at (b), a slip-jump of the size

$$
J=f_{S}-f_{D}
$$




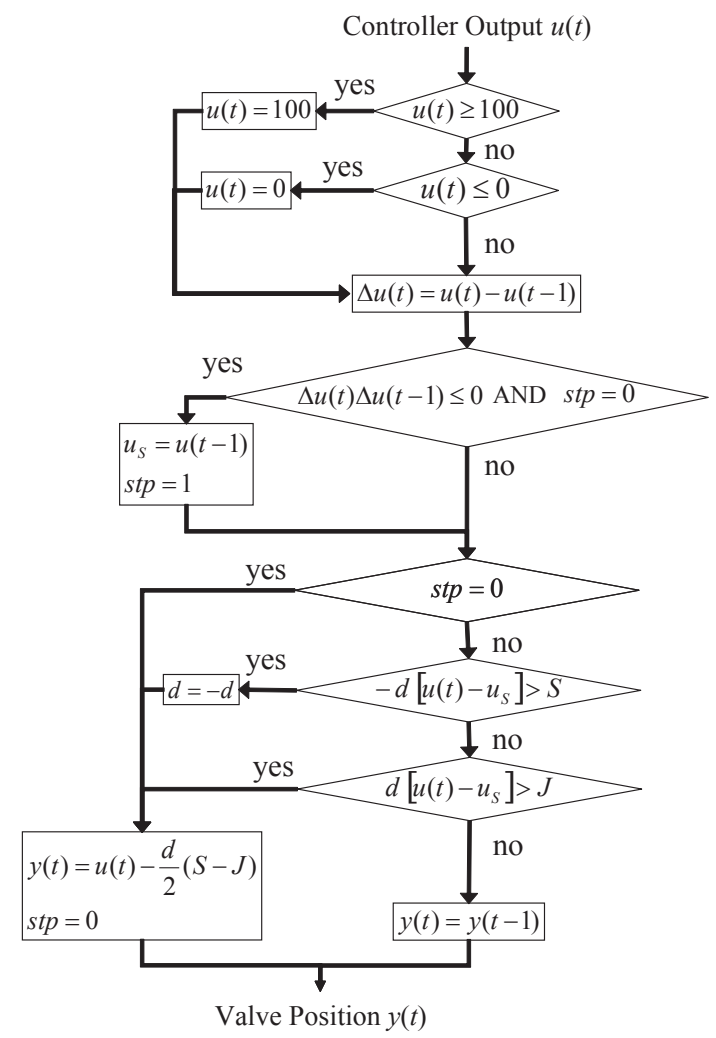

Fig. 3. Flowchart of valve stiction model

happens and the valve state changes from (b) to (c). Thereafter, the valve state changes along the line $l_{2}$ which deviates from the ideal line by $f_{D}$ because the difference between air pressure and elastic force is equal to $f_{D}$. When the valve stops at (d), the difference between air pressure and elastic force needs to exceed $f_{S}$ again for the valve to open further. Since the difference between them is $f_{D}$ at (d), air pressure must increase by $J$ to open the valve. Once air pressure exceeds elastic force by $f_{D}$, the valve state changes to (e) and then follows $l_{2}$.

Air pressure begins to decrease when the controller orders the valve to close at (f). At this moment, the valve changes its direction and comes to rest momentarily. The valve position does not change until the difference between elastic force and air pressure exceeds the maximum static frictional force $f_{S}$. The valve state $(\mathrm{h})$ is just point-symmetric to (b). The difference of air pressure between (f) and (h) is given by

$$
S=f_{S}+f_{D} .
$$

The valve state follows the line $l_{1}$ while the valve position decreases.

The above-mentioned phenomena can be modeled as a flowchart shown in Fig. 3. The input and output of this valve stiction model are the controller output $u$ and the valve position $y$, respectively. Here, the controller output is

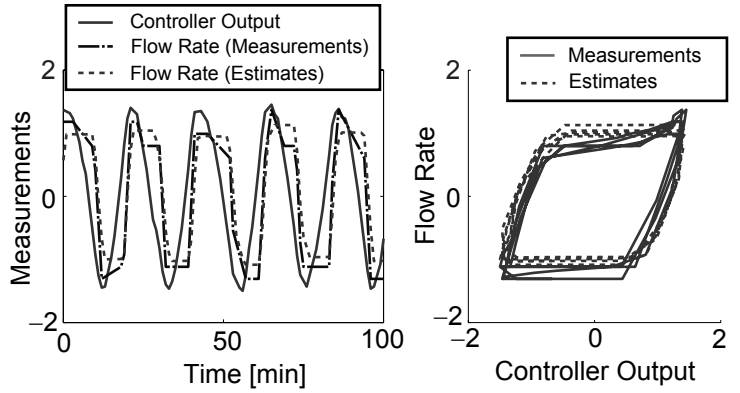

Fig. 4. Comparison between measurements and estimates of flow rate

transformed to the range corresponding to the valve position in advance.

The first two branches check if the upper and the lower bounds of the controller output are satisfied. In this model, two states of the valve are explicitly distinguished: 1$)$ a moving state $(s t p=0)$, and $2)$ a resting state $(s t p=1)$. In addition, the controller output at the moment the valve state changes from moving to resting is defined as $u_{S}$. $u_{S}$ is updated and the state is changed to the resting state $(s t p=1)$ only when the valve stops or changes its direction $(\Delta u(t) \Delta u(t-1) \leq 0)$ while its state is moving $(s t p=0)$. Then, the following two conditions concerning the difference between $u(t)$ and $u_{S}$ are checked unless the valve is in a moving state. The first condition judges whether the valve changes its direction and overcomes the maximum static friction (corresponding to (b) and (h) in Fig. 2). Here, $d= \pm 1$ denotes the direction of frictional force. The second condition judges whether the valve moves in the same direction and overcomes friction. If one of these two conditions is satisfied or the valve is in a moving state, the valve position is updated via the following equation.

$$
y(t)=u(t)-d f_{D}=u(t)-\frac{d(S-J)}{2}
$$

On the other hand, the valve position is unchanged if the valve remains in a resting state.

To demonstrate the validity of this stiction model, simulation results are compared with operation data of a chemical process that suffers from valve stiction. The flow rate is estimated by calculating the valve position from the controller output with the stiction model and by assuming dynamics from the valve position to the flow rate is given by a first order model.

$$
P_{F}(t)=\frac{1}{0.2 s+1}
$$

Measurements and estimates of the flow rate are shown together with the controller output in Fig. 4. The flow rate measurements proved coincident with that estimated by the stiction model. That is, the proposed model can describe 
stiction successfully by using only two parameters $S$ and $J$. By changing these two parameters, the stiction model can describe a variety of situations, for example, an ideal situation when $S=J=0$ and no slip-jump when $J=0$.

The valve stiction model developed here has several advantages compared with the model proposed by Choudhury et al. (2004). First, it can cope with stochastic input as well as deterministic input. Second, $u_{S}$ can be updated at appropriate timings by introducing the valve state $s t p$. Third, it can change the degree of stiction according to the direction of the valve movement.

\section{STICTION DETECTION METHODS}

In this section, two new methods for detecting valve stiction are proposed.

As shown in Fig. 2, the following characteristics are observed when stiction occurs.

(1) There are sections where the valve position does not change even though the controller output changes. Stiction is stronger as such sections are longer.

(2) The relationship between the controller output and the valve position takes the shape of a parallelogram if slip-jump $J$ is neglected. Stiction is stronger as the distance between $l_{1}$ and $l_{2}$ is longer.

On the basis of these characteristics, new methods for detecting valve stiction are proposed. The first method, referred to as method A, is based on characteristic (1); and the second, method B, is based on characteristic (2).

The algorithm of method $\mathrm{A}$ is summarized as follows:

(1) Calculate the difference of valve position y.

$$
\Delta y(t)=y(t)-y(t-1)
$$

(2) Find time intervals when the following condition is satisfied.

$$
|\Delta y(t)|<\varepsilon
$$

where $\varepsilon$ is a threshold.

(3) During each time interval found, calculate the difference between the maximum and the minimum of the controller output $u$ and define it as $\tilde{u}$. Similarly, calculate the difference between the maximum and the minimum of the valve position $y$ and define it as $\tilde{y}$. In addition, determine thresholds: $\varepsilon_{u}$ for $\tilde{u}$ and $\varepsilon_{y}$ for $\tilde{y}$.

(4) Conclude that stiction occurs when $\tilde{u} \geq \varepsilon_{u}$ and $\tilde{y} \leq \varepsilon_{y}$. Otherwise, conclude that stiction does not occur.
(5) Calculate the ratio $\rho$ of total length of intervals when stiction occurs to total length of all intervals. In addition, calculate $\sigma$ that is the mean of $\tilde{u}$ when stiction occurs.

There is higher possibility of stiction as the normalized measure $\rho$ is closer to one. On the contrary, it is confirmed that no stiction occurs when $\rho$ is zero. Furthermore, the degree of stiction can be quantified by using $\sigma$.

Method B is based on the fact that the relationship between the controller output and the valve position takes the shape of a parallelogram and the distance between $l_{1}$ and $l_{2}$ increases as stiction becomes stronger. To capture the difference of shapes, a function $F$ is introduced.

$$
F(t)=\max \left\{\min \left\{F(t-1)+\Delta u(t), F_{\max }\right\}, 0\right\}(7)
$$

The function $F$ indicates the difference between the controller output $u$ and the value on the line $l_{1}$ at the same valve position. Therefore, $u-$ $F$ will have a strong correlation with the valve position $y$. In addition, the maximum value $F_{\max }$ of $F$ corresponds to $S-J$. Thus, the degree of stiction or deadband can be quantified by $F_{\max }$ because slip-jump $J$ is much smaller than deadband $S$ and $F_{\max }$ is usually close to $S$. This is the most important advantage of using method B. To date, no stiction detection method that enables one to quantify stiction has been proposed. $F_{\max }$ and its initial value $F(0)$ can be identified from operation data by solving an optimization problem that aims to maximize a correlation coefficient $r$ between $u-F$ and $y$. As a result, the possibility of stiction gets larger as $F_{\max }$ becomes larger. However, it should be noted here that results of method $\mathrm{B}$ are reliable only when the correlation coefficient $r$ is close to one.

\section{APPLICATIONS}

In this section, the proposed valve stiction detection methods, $\mathrm{A}$ and $\mathrm{B}$, as well as a conventional method are applied to simulation data generated by using the stiction model and operation data obtained from several chemical processes; the usefulness of the proposed methods is also demonstrated.

\subsection{Simulation}

A flow control system and a level control system are investigated here. Block diagrams of both control systems are shown in Fig. 5. Process transfer functions of flow $P_{F}(s)$ and level $P_{L}(s)$ are given by 
Flow Control System

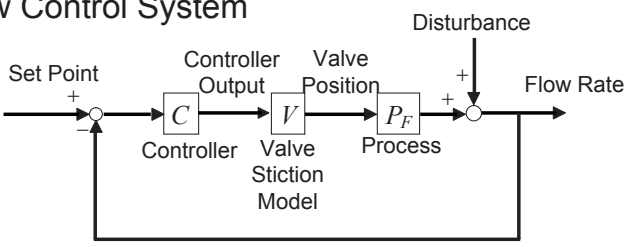

Level Control System

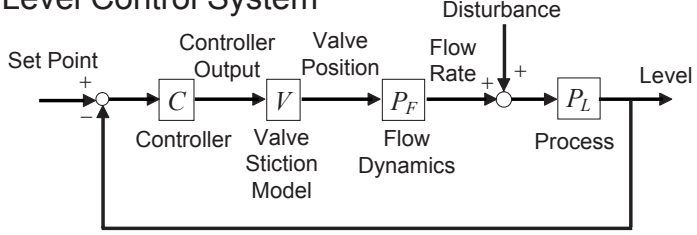

Fig. 5. Block diagram of control systems

Table 1. Controller tuning

\begin{tabular}{ccc}
\hline & Propotional Gain & Integral Time [min] \\
\hline Flow Control & 0.5 & 0.3 \\
Level Control & 3 & 30 \\
\hline
\end{tabular}

Table 2. Parameters of stiction model

\begin{tabular}{lcc}
\hline & $S[\%]$ & $J[\%]$ \\
\hline Case 1 (No Stiction) & 0 & 0 \\
Case 2 (Weak Stiction) & 1 & 0.3 \\
Case 3 (Strong Stiction) & 5 & 1 \\
\hline
\end{tabular}

Table 3. Application results

\begin{tabular}{lccccc}
\hline \multicolumn{5}{c}{$\mathrm{A}$} & \multicolumn{2}{c}{$\mathrm{B}$} & $\mathrm{H}$ \\
\multicolumn{7}{c}{$\rho$} & $\sigma$ & $F_{\max }$ & $r$ & Stiction \\
\hline Flow Control (FC) \\
Case 1 & 0.00 & 0.00 & 0.00 & 0.03 & Yes \\
Case 2 & 0.77 & 0.60 & 0.00 & 0.18 & Yes \\
Case 3 & 0.83 & 3.50 & 0.00 & 0.11 & Yes \\
\hline Level Control - F (LC-F) \\
Case 1 & 0.00 & 0.00 & 0.00 & 1.00 & Yes \\
Case 2 & 0.56 & 0.83 & 0.74 & 1.00 & Yes \\
Case 3 & 0.79 & 4.54 & 4.20 & 0.99 & Yes \\
\hline Level Control - L (LC-L) & & \\
Case 1 & 0.05 & 0.54 & 0.00 & 0.53 & Yes \\
Case 2 & 0.02 & 0.68 & 0.00 & 0.65 & Yes \\
Case 3 & 0.00 & 0.00 & 0.00 & 0.82 & Yes \\
\hline \multicolumn{7}{c}{}
\end{tabular}

$$
P_{F}(s)=\frac{1}{0.2 s+1} \quad, \quad P_{L}(s)=\frac{1}{15 s} e^{-s}
$$

where the unit of time is min. PI controllers are used for both control systems. Control parameters and valve stiction model parameters are summarized in Tables 1 and 2, respectively. Simulation results are shown in Figs. 6 and 7 . Three methods are applied to normalized data of 300 samples. The results are summarized in Table 3. Here, $\mathrm{H}$ denotes the method proposed by Horch (2000). Method H assumes persistent fluctuation of the controlled variables, and its judgment is based on the probability distribution of the controlled variables. LC-F is the case where flow rate is used for detection. On the other hand, LC-L is the case where level is used. Average computational times of methods $\mathrm{A}, \mathrm{B}$, and $\mathrm{H}$ are $0.07 \mathrm{sec}, 1.12 \mathrm{sec}$, and $79 \mathrm{sec}$, respectively. The computational load of method
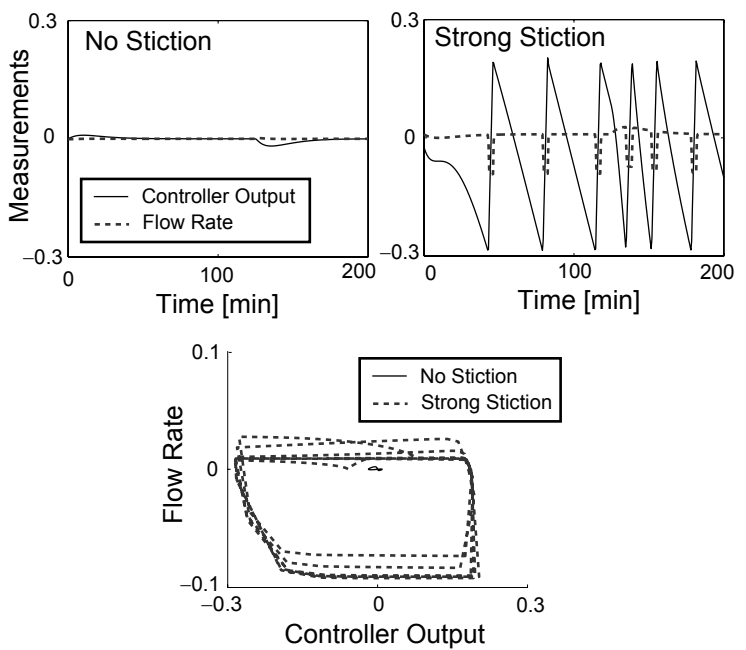

Fig. 6. Simulation results of flow control system
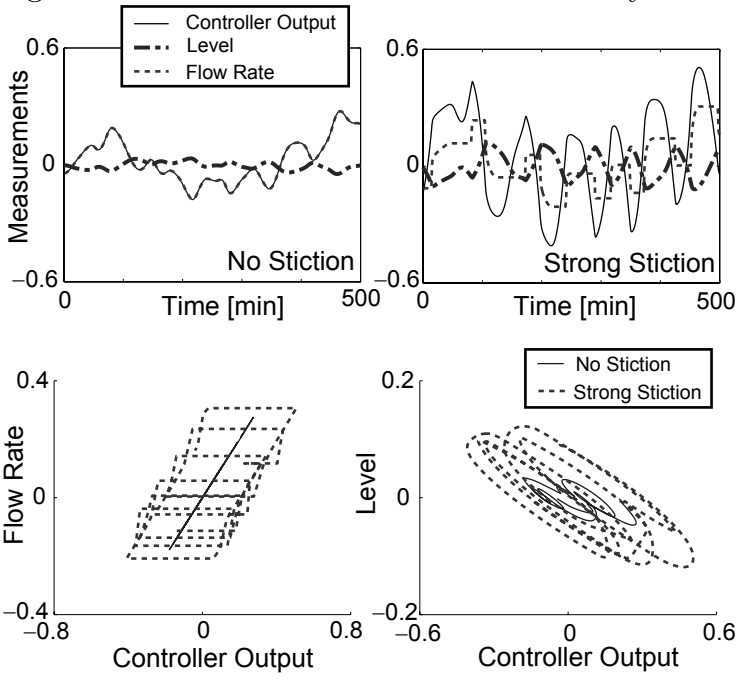

Fig. 7. Simulation results of level control system

$\mathrm{H}$ is excessively heavy, because it requires solving two optimization problems to fit data into two predefined probability distributions.

In case FC, only method A can detect stiction successfully. Method B fails because the relationship between the controller output and the flow rate does not take the shape of a parallelogram. Method $\mathrm{H}$ concludes that stiction occurs in all cases. Of course, this is not true. Method $\mathrm{H}$ does not function well in case 1 because flow rate does not fluctuate persistently. In case LC-F, methods A and B can detect stiction successfully. In addition, the degree of stiction was estimated from $\sigma$ or $F_{\max }$ in each case. These values proved coincident with those in Table 2 . That is, the proposed methods can detect valve stiction and also quantify the stiction, but method $\mathrm{H}$ does not function well. In case LC-L, no method can detect stiction successfully. Methods A and $\mathrm{B}$ do not function well in such a case where the controlled variable is delayed. Therefore, these methods should be used only when flow rate or valve position is measured. Method $\mathrm{H}$ is applicable 
Table 4. Application results

\begin{tabular}{llccccc}
\hline & & \multicolumn{2}{c}{$\mathrm{A}$} & \multicolumn{2}{c}{$\mathrm{B}$} & Horch \\
\multicolumn{2}{l}{ Case } & $\rho$ & $\sigma$ & $F_{\max }$ & $r$ & Stiction \\
\hline 1 & LC (LC-F) & 0.50 & 1.72 & 2.12 & 0.98 & Yes \\
2 & FC & 0.31 & 1.21 & 1.92 & 0.97 & Yes \\
3 & LC (LC-F) & 0.13 & 0.89 & 0.17 & 0.95 & Yes \\
4 & FC & 0.03 & 0.80 & 0.00 & 0.13 & Yes \\
\hline
\end{tabular}

only when persistent fluctuation is found in a controlled variable.

\subsection{Operation Data from Chemical Processes}

Normalized operation data obtained from four chemical processes are shown in Fig. 8. The results of applying three methods to the data are summarized in Table 4 . In cases 1 and 2, all methods can detect stiction successfully. In case 3 , where there is no stiction but bad controller tuning causes fluctuation, method $\mathrm{H}$ incorrectly concludes that there is stiction. However, methods $\mathrm{A}$ and $\mathrm{B}$ give very small indexes $\rho$ and $F_{\max }$, respectively, that is, methods $\mathrm{A}$ and $\mathrm{B}$ correctly conclude that there is no stiction. In case 4 where no stiction occurs, method $\mathrm{H}$ reaches a wrong conclusion again. Method A does, however, reach the right conclusion. Although method B reaches the right conclusion, the correlation coefficient $r$ is too small and thus the result is not reliable.

\section{CONCLUSIONS}

In the present work, a valve stiction model is developed and new stiction detection algorithms are proposed. The validity of this model is demonstrated by comparing simulation results

Case 1 : Level Control - Valve Stiction
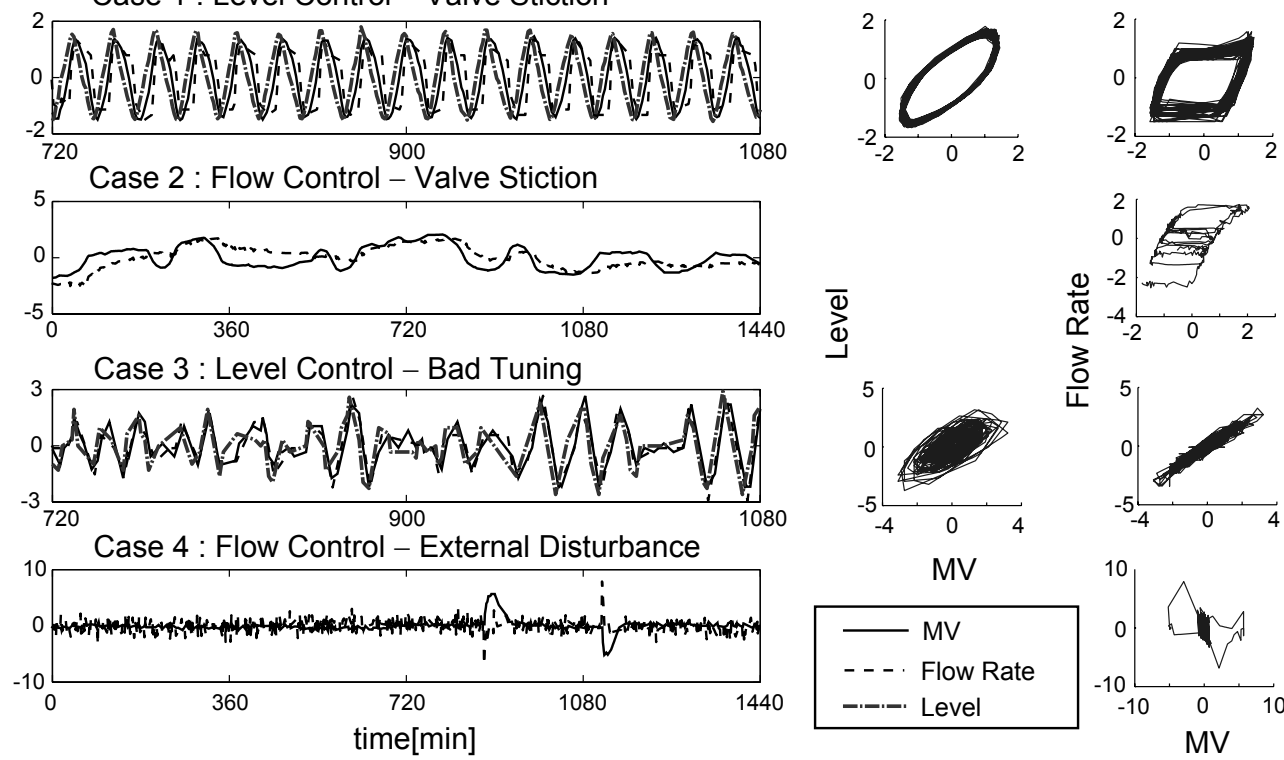

This research is partially supported by the consortium No.25 organized in the PSE 143th comittee of JSPS.

\section{REFERENCES}

Choudhury, M.A.A.S., N.F. Thornhill, and S.L. Shah (2004). A Data-Driven Model for Valve Stiction. IFAC Symposium on Advanced Control of Chemical Processes (ADCHEM), Hong-Kong, Jan. 11-14.

Horch, A. (2000). Condition Monitoring of Control Loops. PhD thesis, Royal Institute of Technology, Stockholm, Sweden.

Muller, F. (1994). Simulation of an Air Operated Sticky Flow Control Valve. Proc. of the 1994 Summer Computer Simulation Conference, 742-745.

\section{Ð}
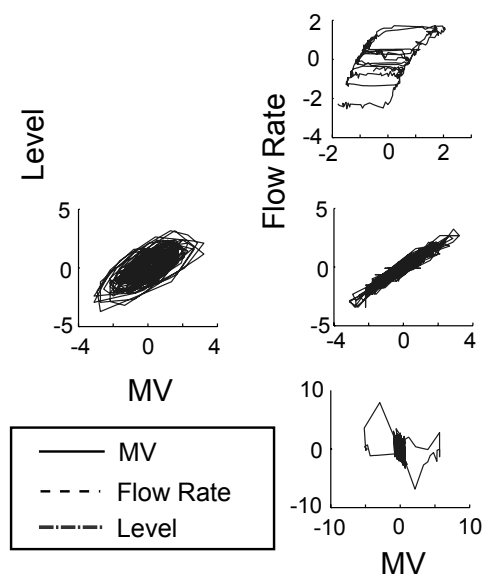

Fig. 8. Operation data of four control systems 